Universidad Nacional Experimental Francisco de Miranda (UNEFM). Santa Ana de Coro. Venezuela

Mayra Priscila Cáceres-Cabrera; Darwin Gabriel García-Herrera; Nancy Marcela Cárdenas-Cordero Juan Carlos Erazo Álvarez

DOI $10.35381 / \mathrm{cm} . v 6 \mathrm{i} 3.409$

\title{
Juegos tradicionales como estrategia metodológica para la enseñanza de matemática
}

\section{Traditional games as a methodological strategy for teaching mathematics}

\author{
Mayra Priscila Cáceres-Cabrera \\ mayra.caceres.65@est.ucacue.edu.ec \\ Universidad Católica de Cuenca, Azogues \\ Ecuador \\ https://orcid.org/0000-0001-6472-193X \\ Darwin Gabriel García-Herrera \\ dggarciah@ucacue.edu.ec \\ Universidad Católica de Cuenca, Azogues \\ Ecuador \\ https://orcid.org/0000-0001-6813-8100 \\ Nancy Marcela Cárdenas-Cordero \\ ncarenasc@ucacue.edu.ec \\ Universidad Católica de Cuenca, Cuenca \\ Ecuador \\ https://orcid.org/0000-0002-6250-6504 \\ Juan Carlos Erazo Álvarez \\ jcerazo@ucacue.edu.ec \\ Universidad Católica de Cuenca, Cuenca \\ Ecuador \\ https://orcid.org/0000-0001-6480-2270
}

Recibido: 20 de agosto de 2020 Aprobado: 15 de noviembre de 2020 
Universidad Nacional Experimental Francisco de Miranda (UNEFM). Santa Ana de Coro. Venezuela

Mayra Priscila Cáceres-Cabrera; Darwin Gabriel García-Herrera; Nancy Marcela Cárdenas-Cordero Juan Carlos Erazo Álvarez

\title{
RESUMEN
}

En este sentido, el objetivo del presente estudio fue analizar en qué medida las estrategias lúdicas (juegos tradicionales) pueden fortalecer las competencias matemáticas tempranas en los estudiantes de inicial y preparatoria. El presente estudio de tipo no experimental cuyo enfoque epistemológico fue cuantitativo y de corte transversal, tomando como población a los docentes de la red de inicial y preparatoria del Circuito 2 del cantón Paute, con la investigación se obtuvo un resultado en donde se demostró que los docentes aplican juegos tradicionales como una actividad lúdica y no como una estrategia de aprendizaje significativo en la matemática. La falta de implementación de estrategias lúdicas dentro del aprendizaje por parte de los docentes origina aburrimietno en los estudiantes y no sienten motivación por aprender de manera significativa.

Descriptores: Costumbres y tradiciones; educación cultural; método de aprendizaje. (Palabras tomadas del Tesauro UNESCO).

\begin{abstract}
In this sense, the objective of the present study was to analyze to what extent recreational strategies (traditional games) can strengthen early mathematical competencies in kindergarten and high school students. The present non-experimental study whose epistemological approach was quantitative and cross-sectional, taking as population the teachers of the initial and preparatory network of Circuit 2 of the Paute canton, with the investigation a result was obtained where it was demonstrated that the Teachers apply traditional games as a playful activity and not as a meaningful learning strategy in mathematics. The lack of implementation of playful strategies within learning by teachers causes boredom in students and they do not feel motivated to learn in a meaningful way.

Descriptors: Customs and traditions; cultural education; learning methods. (Words taken from the UNESCO Thesaurus).
\end{abstract}


Universidad Nacional Experimental Francisco de Miranda (UNEFM). Santa Ana de Coro. Venezuela

Mayra Priscila Cáceres-Cabrera; Darwin Gabriel García-Herrera; Nancy Marcela Cárdenas-Cordero Juan Carlos Erazo Álvarez

\section{INTRODUCCIÓN}

El juego ocupa una parte primordial dentro de la infancia y la falta de aplicación de estrategias lúdicas por parte de los docentes hace que las clases sean menos creativas y más aburridas, dejando de lado una forma más interesante de enseñar la matemática. Es por eso necesario que los docentes implementen nuevas formas de enseñanza y más aún en la infancia, por cuanto a esta edad es muy importante desarrollar las diferentes destrezas en los niños del nivel preparatoria, evitando llevarles al fracaso escolar en los años posteriores.

En la actualidad mucho de estos fracasos se da porque los estudiantes no adquieren las Competencias Matemáticas necesarias y van perdiendo el interés por aprender, pues los docentes siguen empleando estrategias tradicionales que muchas de las veces no ayudan a que los estudiantes obtengan un aprendizaje significativo, es por esa razón que se debe cambiar estrategias que contribuyan y despierten el interés de los estudiantes.

De acuerdo a los estudios realizados por las pruebas PISA-D (Programa para la Evaluación Internacional de Estudiantes) efectuado en 2018 en Ecuador, el 70,8\% de los estudiantes no alcanzaron al nivel 2 de matemáticas obteniendo un bajo desempeño en esta área, reflejando una educación memorista que no ayuda al estudiante en el uso de la lógica para la resolución de problemas (El Universo, 2019). Es por eso necesario realizar una investigación para identificar la contribución que nos brinda los juegos en niños y niñas que cursan los primeros años de primaria ofreciendo así la posibilidad de prevenir dificultades en su aprendizaje.

Es muy importante el papel que cumple el docente en la formación intelectual de los niños y por esta razón debe centrarse en aplicar actividades lúdicas para poder desarrollar competencias matemáticas que ayuden al estudiante a desenvolverse mejor a lo largo de su vida (Zafra, Vergel , \& Martínez, 2016). 
Universidad Nacional Experimental Francisco de Miranda (UNEFM). Santa Ana de Coro. Venezuela

Mayra Priscila Cáceres-Cabrera; Darwin Gabriel García-Herrera; Nancy Marcela Cárdenas-Cordero Juan Carlos Erazo Álvarez

Aplicar los juegos tradicionales como una estrategia metodológica en el aprendizaje de los estudiantes en edades tempranas favorecerá su atención, seguridad, creatividad para buscar solución a sus dificultades,

Para el desarrollo de la investigación se planteó la siguiente pregunta: ¿En qué medida el uso de los juegos tradicionales contribuye a fortalecer las competencias matemáticas en los niños y niñas del nivel inicial y preparatoria? La misma que guiará la consecución del objetivo de la investigación: Analizar en qué medida las estrategias lúdicas (juegos tradicionales) pueden fortalecer las competencias matemáticas tempranas en los estudiantes del nivel de inicial y preparatoria.

\section{Referencia teórico}

Para abordar este tema se revisó varios trabajos de investigación de diferentes autores en donde se pudo seleccionar algunos que nos dan soporte teórico a nuestro tema de investigación. Varias de ellas ayudaron a confirmar que la aplicación de las estrategias lúdicas (juegos tradicionales), además de ser una diversión, aportan de manera muy significativa en el proceso educativo del niño. A continuación, se examinarán algunos de ellos:

El uso de las actividades Lúdicas son la base importante en la enseñanza de la matemática en edades tempranas, es por eso que (Quintanilla, 20016) realizo una investigación no experimental sobre las actividades lúdicas a los docentes de la Escuela "Profesora Teresa de Jesús" en donde al aplicar las debidas encuestas y entrevistas pudo evidenciar que el $83 \%$ de los docentes no aplican actividades lúdicas para la enseñanza y esto se da por falta de desconocimiento de Estrategias a pesar de que ellos consideran importante el juego en los estudiantes para el aprendizaje.

Según ( Ferreira \& Sousa, 2017), dentro de su trabajo de investigación realizada en base a los juegos para enseñar la matemática, aplicando diferentes técnicas e instrumentos de investigación les permitió identificar la importancia que tiene implementar actividades 
Universidad Nacional Experimental Francisco de Miranda (UNEFM). Santa Ana de Coro. Venezuela

Mayra Priscila Cáceres-Cabrera; Darwin Gabriel García-Herrera; Nancy Marcela Cárdenas-Cordero Juan Carlos Erazo Álvarez

lúdicas en la enseñanza de los niños en la primera infancia pues el estudio realizado a los docentes de la escuela Mato Grosso de Brasil pudieron evidenciar que no se logra desarrollar aprendizajes de forma significativas, ya que los docentes imparten sus enseñanzas de forma superficial requiriendo un cambio en la metodología de los docentes y fortaleciendo el aprendizaje de los estudiantes.

(Awalludin, Handoyo, \& Sri, 2018), al realizar su investigación sobre el efecto del aprendizaje con el uso del juego tradicional basándose en el método cuantitativo y con grupo de control y un grupo experimental, demostraron que hay una gran diferencia de manera muy significativa en el aprendizaje de los estudiantes, mejorando incluso su habilidad social. Por otro lado, los autores ( González , Juan , \& Sánchez ,2014) al realizar una revisión literaria de varias investigaciones sobre los efectos del uso del juego en la enseñanza, expresan que prevalece el entusiasmo y aspectos positivos en la enseñanza de la matemática en muchos de los docentes mediante juegos, pero hace falta más motivación e implementación de guías lúdicas dentro de las instituciones para que puedan ser implementadas y se pueda demostrar resultados más sólidos.

Dentro de la investigación de (Aristizábal , Colorado, \& Gutiérrez , 2016), durante su estudio indican que al implementar varias estrategias lúdicas a los estudiantes evidenciaron que el grupo experimental alcanzo un porcentaje más alto en la eficiencia de las matemáticas que el grupo que trabajaba con una metodología tradicional, concluyendo así que el juego en la infancia contribuye de manera muy significativa al conocimiento matemático.

También (Fernández, Molina, \& Oliveras, 2016) implementaron su investigación- acción en un centro de educación infantil con una muestra de 25 niños de 4 años de edad, mediante este estudio determinaron la importancia de implementar los juegos para la enseñanza, recordando que esto son algo natural del niño, los resultados obtenidos fueron muy satisfactorios ya que, al implementar juegos de manera organizada y bien planificada, dieron buenos resultados en la adquisición de conocimientos matemáticos, 
Universidad Nacional Experimental Francisco de Miranda (UNEFM). Santa Ana de Coro. Venezuela

Mayra Priscila Cáceres-Cabrera; Darwin Gabriel García-Herrera; Nancy Marcela Cárdenas-Cordero Juan Carlos Erazo Álvarez

manifestando que las personas que dirigen la enseñanza de los niños de esta corta edad deben ser profesionales aptos y preparados para poder obtener una educación de calidad. Como menciona (Condor, 2019), los juegos tradicionales son una gran influencia para el aprendizaje en los niños y niñas de 6 a 7 años en el desarrollo del pensamiento lógico, y para su estudio utilizaron una metodología de tipo cualitativo y cuantitativo, tomando dos grupos de estudiantes, el grupo control y el experimental con una muestra de 13 y 14 estudiantes en donde obtuvieron resultados favorables y positivos mejorando el aprendizaje matemático.

De igual manera (Putu \& Prima, 2017) en su trabajo de investigación sobre los juegos tradicionales en la primera infancia socioemocional realizado en los estudiantes del B TK Pradnyandari III Kerobokan del año académico 2016- 2017 utilizando el método de observación, manifiestan que los estudiantes mejoraron sus habilidades socioemocionales, evidenciando que existe una relación muy importante entre los juegos tradicionales y el logro del aprendizaje de los niños en cualquier área que podamos aplicar.

Por otra parte se presenta a (Sánchez, 2019) quien en su investigación sobre las Estrategias Lúdicas en el aprendizaje, empleando una metodología cualitativa y entrevistas, pudo verificar que muchos de los estudiantes no alcanzaban a desarrollar nociones básicas necesarias en el ámbito de la matemática, al igual que las docentes no empleaban estrategias lúdicas para motivar y enseñar en sus clases, recalcando así la importancia de los juegos para interiorizar los aprendizajes en los niños y niñas. Mientras que (Caballero \& Espínola, 2016), en su análisis realizado en la ciudad de México basándose en los resultados del ciclo escolar 2009 - 2014 en el área de matemáticas los estudiantes de bachillerato no han impactado de forma significativa en el desarrollo de las habilidades, pues adquieren ciertos conocimientos matemáticos desde el nivel preescolar hasta los niveles superiores de forma superficial, memorística haciendo que al final los estudiantes definan carreras que no contengan matemáticas, 
Universidad Nacional Experimental Francisco de Miranda (UNEFM). Santa Ana de Coro. Venezuela

Mayra Priscila Cáceres-Cabrera; Darwin Gabriel García-Herrera; Nancy Marcela Cárdenas-Cordero Juan Carlos Erazo Álvarez

dándose este problema por la falta de aplicación de actividades lúdicas y el maltrato por parte de las docentes desmotivando así a los estudiantes puesto que no llegan a desarrollar las nociones básicas que se requiere.

Las estrategias lúdicas agrupan varias actividades planificadas, organizadas y con una orientación a la construcción del aprendizaje de una manera divertida, con el objetivo de mejorar la enseñanza; los docentes deben implementar estrategias encaminadas hacia un aprendizaje renovado y significativo, dejando de lado lo tradicional, ser puestas en práctica con la finalidad que los niños interioricen con profundidad los saberes por mucho más tiempo, (Sánchez, 2019).

El juego es parte importante dentro del aprendizaje de los niños, (Minerva, 2002) manifiesta que dentro de la enseñanza el juego es considerado como un método que ayuda a los estudiantes a alcanzar conocimientos en diferentes ámbitos al mismo tiempo crea satisfacción al ser entretenido y divertido, razón por la cual el espacio educativo debe llevar a cabo actividades lúdicas para poder llegar a los estudiantes y desarrollar de mejor manera los aprendizajes requeridos. Muchas de las veces los docentes de niños y niñas se olvidan que el estudiante a temprana edad necesita gran parte de su tiempo para el juego, al igual que (Irvin , 2017) manifiesta que el juego es parte esencial de la niñez ya que ayuda a adquirir habilidades importantes para su vida diaria favoreciendo así su desarrollo hasta su edad adulta.

Los autores (Sailema \& Sailema) determinan que el juego "es un conjunto de actividades que nos ponen en manifiesto con las experiencias, vivencias y el conocimiento" (p. 21), es decir, son acciones que al realizarlas ayudará a los estudiantes a involucrarse en situaciones que vivimos diariamente y de ellas van adquiriendo conocimientos o habilidades que les permitirá desenvolverse de mejor manera en su entorno.

Los juegos tradicionales son parte de las actividades lúdicas, (Morera ,2008) indica que han venido trascendiendo de tiempos muy lejanos y llevándose a cabo de generación en generación, con los cuales jugaron muchos de nuestros abuelos, padres y hermanos, son 
Universidad Nacional Experimental Francisco de Miranda (UNEFM). Santa Ana de Coro. Venezuela

Mayra Priscila Cáceres-Cabrera; Darwin Gabriel García-Herrera; Nancy Marcela Cárdenas-Cordero Juan Carlos Erazo Álvarez

considerados como una costumbre de un pueblo, se transmiten de forma oral, van apareciendo por épocas con riesgo de perderse y muchas de las veces van realizándose cambios de acuerdo a la necesidad o época en la que se encuentra, y en su mayoría son practicados a nivel mundial y en su mayoría no se necesita de implementos tecnológicos para poder desarrollarlos y es una estrategia divertida que se puede implementar dentro de las instituciones, para mejorar la enseñanza y aprendizaje de los estudiantes.

Vale la pena indicar que ( Perugachi , 2018) señala que el juego es una parte primordial dentro del desarrollo del ser humano sin distinción de edad, género o etnia, y cabe destacar que Ecuador es un país pluricultural y multiétnico que abarca diversos pueblos con identidad propia, y cada uno de los ellos desarrollan sus juegos tradicionales de acuerdo a su cultura e identidad, adaptándolas con sus propias normas, reglas, participantes.

Existen diferentes juegos tradicionales, (Mendoza, Analuiza, \& Lara, 2017) dan a conocer algunos como: el trompo, las canicas, los ensaquillados, la gallina ciega, la cuchara y el huevo, el juego de la silla, las rondas, la rayuela, entre otros muchos de ellos pueden ser dirigidos por los mismos niños sin la necesidad de una persona adulta lo cual ayuda a desarrollar independencia personal para desenvolverse en un medio social. Punto importante que indica (Öfele, 2014) es que los pilares fundamentales de los niños son: el juego, la ternura y el encuentro, y cada uno de estos pilares al ser trabajado de manera correcta ayudará al niño a tener estabilidad emocional, pues al crear confianza él niño demostrará esfuerzo en desarrollar sus habilidades sin tener que buscar a cada instante el consentimiento de otra persona. Hay varios beneficios que brindan la práctica de los juegos tradicionales, (Restrepo \& Tamayo , 2017) dan a conocer algunos de ellos a continuación: 
Universidad Nacional Experimental Francisco de Miranda (UNEFM). Santa Ana de Coro. Venezuela

Mayra Priscila Cáceres-Cabrera; Darwin Gabriel García-Herrera; Nancy Marcela Cárdenas-Cordero Juan Carlos Erazo Álvarez

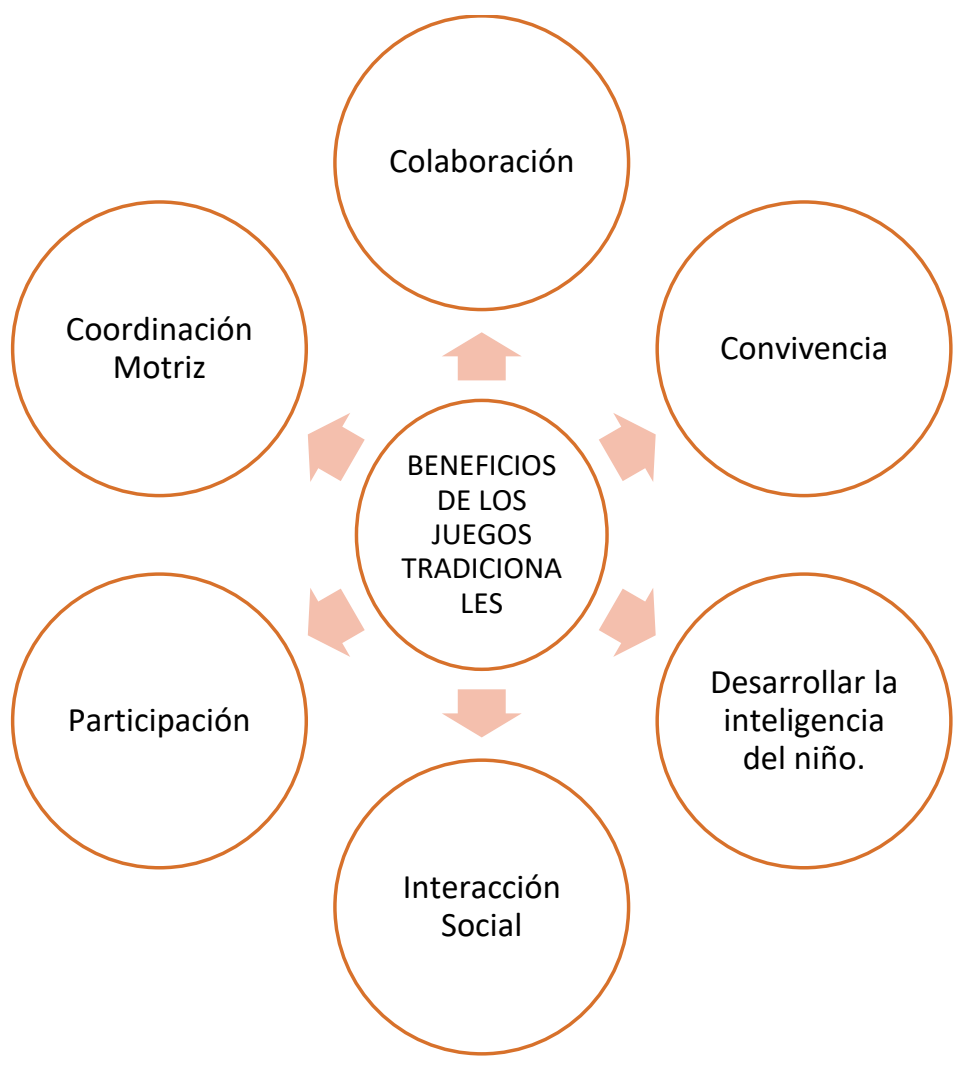

Figura 1. Beneficios de los juegos tradicionales en el niño.

Fuente: Adaptado de Restrepo \& Tamayo (2017).

La matemática es una de las materias importantes que ayuda al desarrollar el pensamiento lógico, mediante la aplicación de algunas fases como: la observación, clasificación, comparación, sin embargo, es muy compleja para ser abordada por el estudiante, no es una tarea fácil llegar a la compresión de los contenidos, es por eso indispensable implementar las herramientas precisas y sobre todo entretenidas que motiven hasta que los estudiantes adquieran las habilidades y destrezas necesarias (Moya , 2004).

La motivación es una actitud positiva de las personas que se interesan por aprender a realizar alguna actividad (Carrillo, Padilla, Rosero, \& Villagómez, 2009). Parte importante dentro de la enseñanza es la motivación de los docentes hacia los estudiantes ya que es 
Universidad Nacional Experimental Francisco de Miranda (UNEFM). Santa Ana de Coro. Venezuela

Mayra Priscila Cáceres-Cabrera; Darwin Gabriel García-Herrera; Nancy Marcela Cárdenas-Cordero Juan Carlos Erazo Álvarez

el motor que incentiva a potencializar el aprendizaje (Ospina , 2006). El docente está en la obligación de cambiar el aprendizaje, generando ideas innovadoras para que el estudiante tenga una participación activa y llegar a obtener el objetivo planteado, y para eso debe tener un alto nivel de motivación. (Garrote, Garrote, \& Jiménez, 2016).

Por consiguiente al implementar Actividades lúdicas (juegos tradicionales) en la enseñanza de las matemáticas en los niños del subnivel preparatoria, motivará a desarrollaran de manera significativa los conocimientos, puesto que lo harán de manera divertida, por esta razón, (Aristizábal, Colorado, \& Gutiérrez, 2016), determinan que estos juegos proporciona en los estudiantes liderazgo, integración, inclusive indagan estrategias para solucionar desafíos planteados dentro del juego, desarrollando así competencias intelectuales, sociales, emocionales y es allí donde los docentes deben aprovechar al máximo promoviendo la búsqueda del conocimiento.

\section{METODOLOGÍA}

Para la presente investigación de tipo descriptiva no experimental, cuantitativo y de corte transversal puesto que se realizó en un solo tiempo, se tomó como población a 28 docentes de la red de inicial y preparatoria del Circuito 2 del cantón Paute, de los cuales se asumió una muestra de 23 docentes, en donde se decidió aplicar una encuesta, para la recolección de datos, este instrumento fue elaborado en Google Forms, la misma que fue validada a través del análisis de fiabilidad del programa IBM SPSS con un valor aceptable de 0,75, obteniendo así información a través de las gráficas y resultados estadísticos. 
CIENCIAMATRIA

Revista Interdisciplinaria de Humanidades, Educación, Ciencia y Tecnología

Año VI. Vol. VI. N³. Edición Especial III. 2020

Hecho el depósito de ley: pp201602FA4721

ISSN-L: 2542-3029; ISSN: 2610-802X

Universidad Nacional Experimental Francisco de Miranda (UNEFM). Santa Ana de Coro. Venezuela

Mayra Priscila Cáceres-Cabrera; Darwin Gabriel García-Herrera; Nancy Marcela Cárdenas-Cordero Juan Carlos Erazo Álvarez

\section{RESULTADOS}

Los resultados se obtuvieron mediante el análisis de la normalidad de las variables adquiriendo un intervalo de confianza del $95 \%$, y dando como resultado que todas son paramétricas debido a que tiene un valor menor a 0,05. A continuación, se realizará un análisis de los cuadros estadísticos:

\section{Tabla 1.}

Relación entre frecuencias del uso de Juegos tradicionales para la enseñanza de matemática y Como docente de inicial o preparatoria, usted ha implementado juegos tradicionales en sus clases para enseñar matemática.

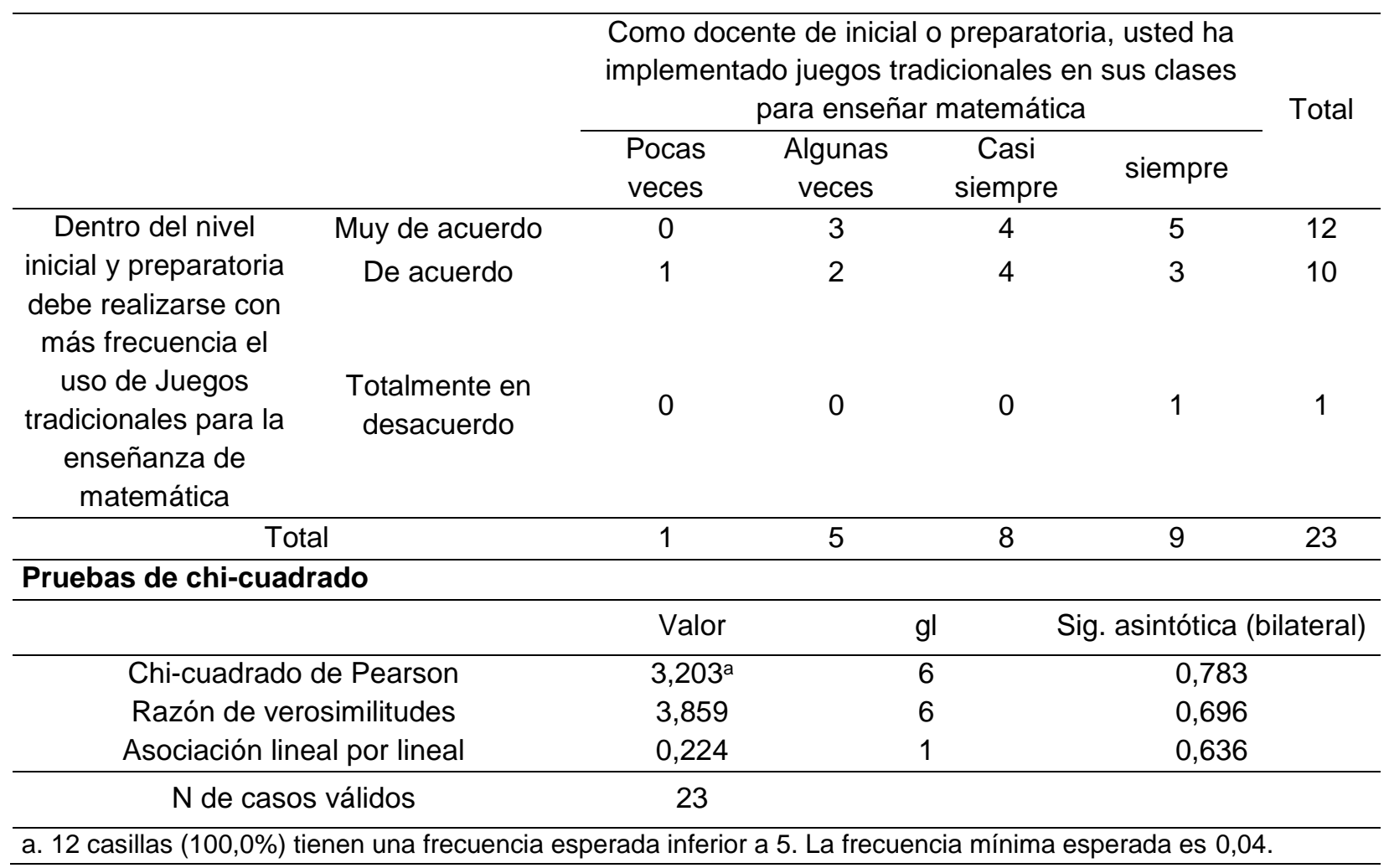

Fuente: Elaboración propia. 
Universidad Nacional Experimental Francisco de Miranda (UNEFM). Santa Ana de Coro. Venezuela

Mayra Priscila Cáceres-Cabrera; Darwin Gabriel García-Herrera; Nancy Marcela Cárdenas-Cordero Juan Carlos Erazo Álvarez

En la tabla 1 poder observar el análisis del chi- cuadrado con un valor de 0,783 siendo mayor a 0,05 determinando de esta manera que son datos no asociados por lo cual se asume que la hipótesis es nula, debido a ello se determina que no hay relación entre la variable que los docentes han implementado juegos tradicionales en sus clases para enseñar matemática y que en estos niveles se deben realizar con más frecuencia el uso de juegos tradicionales en la enseñanza de matemática, de esta manera podemos establecer que los docentes han utilizado estos juegos pero no con tanta frecuencia por tal motivo se establece que se debe en incrementar otros tipos de estrategias con actividades llamativas con los juegos tradicionales para que los estudiantes puedan desarrollar y fortalecer estrategias matemáticas estimulando de manera constante para que los niños y niñas aprendan mientras juegan.

\section{Tabla 2.}

Es importante implementar dentro de las instituciones talleres a los docentes para desarrollar estrategias metodológicas con la implementación de los juegos tradicionales en la enseñanza de la matemática.

\begin{tabular}{cccccc}
\hline & & Frecuencia & Porcentaje & $\begin{array}{c}\text { Porcentaje } \\
\text { válido }\end{array}$ & $\begin{array}{c}\text { Porcentaje } \\
\text { acumulado }\end{array}$ \\
& Muy de acuerdo & 12 & 52,2 & 52,2 & 52,2 \\
\multirow{4}{*}{ Válidos } & De acuerdo & 10 & 43,5 & 43,5 & 95,7 \\
& Totalmente en desacuerdo & 1 & 4,3 & 4,3 & 100,0 \\
\cline { 2 - 6 } & Total & 23 & 100,0 & 100,0 & \\
\hline
\end{tabular}

Fuente. Elaboración propia.

En la tabla 2 se observa que el $95,7 \%$ de los docentes están muy de acuerdo y de acuerdo que dentro de las instituciones se debe implementar talleres para docentes que ayuden a desarrollar estrategias metodológicas con la implementación de los juegos tradicionales para la enseñanza de la matemática en niños y niñas de inicial y preparatoria. Se debe aprovechar de manera correcta los juegos tradicionales en las instituciones educativas es por eso que hace falta que se realice talleres que sirvan como 
Universidad Nacional Experimental Francisco de Miranda (UNEFM). Santa Ana de Coro. Venezuela

Mayra Priscila Cáceres-Cabrera; Darwin Gabriel García-Herrera; Nancy Marcela Cárdenas-Cordero Juan Carlos Erazo Álvarez

una herramienta estratégica que ayude a inmiscuir al aprendizaje en ambientes gratos desarrollando así habilidades en los estudiantes.

\section{Tabla 3}

Los juegos tradicionales ayudan a mejorar el razonamiento matemático en los estudiantes de inicial y preparatoria.

\begin{tabular}{cccccc}
\hline \multirow{2}{*}{ Válidos } & Frecuencia & Porcentaje & Porcentaje válido & $\begin{array}{c}\text { Porcentaje } \\
\text { acumulado }\end{array}$ \\
& & & & & 56,5 \\
& Siempre & 13 & 56,5 & 46,5 \\
\cline { 2 - 6 } & Casi siempre & 10 & 43,5 & 43,5 & 100,0 \\
\cline { 2 - 6 } & Total & 23 & 100,0 & 100,0 & \\
\hline
\end{tabular}

Fuente: Elaboración propia.

En la tabla 3 podemos observar que el 100\% de los docentes están de acuerdo que siempre y casi siempre los juegos tradicionales ayudan a mejorar el razonamiento matemático en los estudiantes de inicial y preparatoria. Es por esa razón que se deben tener espacios de enseñanza en donde los estudiantes disfruten su aprendizaje a través de la participación de los juegos no solo de manera espontánea sino más bien orientando a desarrollar destrezas y habilidades que aporten a sus aprendizajes de forma significativa. 
Universidad Nacional Experimental Francisco de Miranda (UNEFM). Santa Ana de Coro. Venezuela

Mayra Priscila Cáceres-Cabrera; Darwin Gabriel García-Herrera; Nancy Marcela Cárdenas-Cordero Juan Carlos Erazo Álvarez

\section{PROPUESTA}

Luego de haber analizado varias investigaciones bibliográficas y las encuestas realizadas se determina que en su mayoría los docentes manifiestan que los juegos tradicionales ayudan a desarrollar destrezas matemáticas, pero no aplican en dentro de la enseñanza por falta de conocimiento de estrategias didácticas para que puedan ser desarrolladas por los estudiantes.

Es por ese motivo que se ha considerado en realizar algunas actividades con el juego de la rayuela para aplicarlas dentro de matemática en niños y niñas del nivel inicial y preparatoria. De esta manera se establece un proceso que el docente debe llevar acabo para que estos juegos puedan desarrollar destrezas matemáticas en el nivel inicial y preparatoria.

2. Seleccionar juegos de acuerdo a la edad de los

T.Analizar estudiantes.

\section{Evaluar} los

3. Aplicar los juegos con actividades llamativas.

Destrezas a desarrollarse.

Figura 2. Propuesta aplicación Juegos tradicionales.

Fuente: Elaboración propia.

\section{Analizar destrezas que se desea desarrollar en los estudiantes con los juegos} tradicionales. - Los docentes deben analizar las destrezas con Criterio de desempeño más importantes para ser desarrolladas en niños de 4 a 5 años de edad, sustentándose al objetivo que desean alcanzar, con el fin de mejorar su desarrollo cognitivo, habilidades y actitudes que le ayude a desempeñase en su diario vivir. 
Universidad Nacional Experimental Francisco de Miranda (UNEFM). Santa Ana de Coro. Venezuela

Mayra Priscila Cáceres-Cabrera; Darwin Gabriel García-Herrera; Nancy Marcela Cárdenas-Cordero Juan Carlos Erazo Álvarez

Seleccionar juego tradicional de acorde a los estudiantes. - Debe considerarse siempre la edad del niño y niña para seleccionar los juegos, estos deben ser entretenido, de interés y a la vez funcional, es decir, que el niño juegue y aprenda. El juego de la rayuela es uno de los juegos tradicionales que más se presta para la enseñanza de la matemática, es muy divertida y con ella los estudiantes aprenden a desarrollar varias habilidades como reconocer los números, contar, sumar, relacionar, lateralidad, nociones dentro fuera e incluso ayuda a mejorar su equilibrio. Por otro lado, también tenemos el juego de las canicas que ha venido trascendiendo de generación en generación y que es muy interesante para niños y niñas. Pues existe muchas maneras de ponerlas en práctica para enseñar las matemáticas. En el siguiente punto se presentará algunas actividades que se pueden realizar con este juego.

Aplicar juego tradicional con diferentes actividades llamativas. - Antes de comenzar el docente debe dar las indicaciones sobre las reglas del juego y la manera en que la realizará para que el estudiante pueda desenvolverse mejor durante la actividad y la pueda disfrutar mientras aprende.

\section{Reconocimiento del número}

Figura 3. La rayuela.

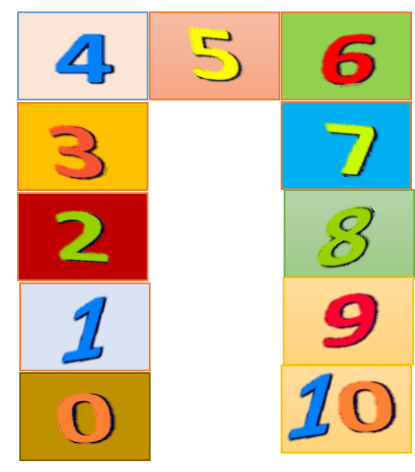

Fuente: Elaboración propia. 
Universidad Nacional Experimental Francisco de Miranda (UNEFM). Santa Ana de Coro. Venezuela

Mayra Priscila Cáceres-Cabrera; Darwin Gabriel García-Herrera; Nancy Marcela Cárdenas-Cordero Juan Carlos Erazo Álvarez

Dibujar una rayuela en el piso y colocar los números del o al 10, el estudiante debe ir saltando dentro de cada casillero nombrando el número y observando su secuencia. También se puede enseñar la secuencia de números de dos en dos, saltando en el $0,2,4,6,8,10$.

\section{Relacionar el número con el numeral - Sumas y retas}
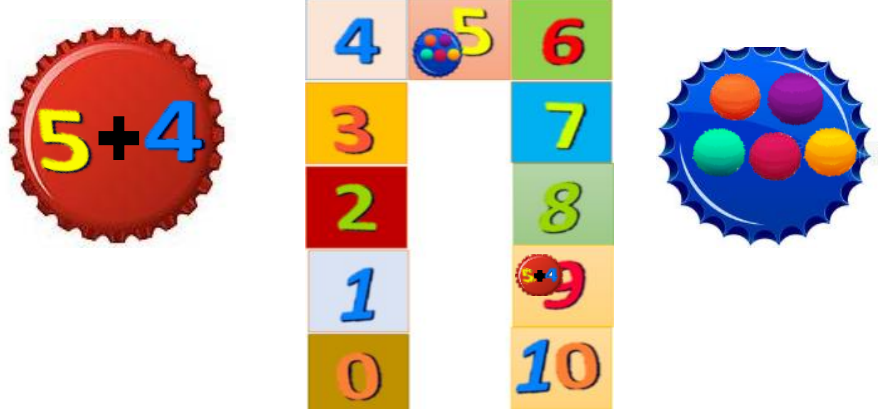

Figura 4. La rayuela y sus elementos.

Fuente. Elaboración propia.

Colocar o dibujar elementos en tapas grande de botella o en algún elemento y el estudiante debe contar los elementos y lanzar al numeral que corresponde.

Para trabajar la suma se coloca en varias tapas las respectivas operaciones y el estudiante debe lanzar a la respuesta que corresponde.

\section{Secuencia numérica ascendente y descendente}

En este juego el estudiante debe ir saltando de forma ascendente con el pie derecho y regresar de forma descendente con el izquierdo, haciendo que repita los números que va pisando, como podemos ver aquí se trabaja incluso lateralidad en los niños y niñas. 
Universidad Nacional Experimental Francisco de Miranda (UNEFM). Santa Ana de Coro. Venezuela

Mayra Priscila Cáceres-Cabrera; Darwin Gabriel García-Herrera; Nancy Marcela Cárdenas-Cordero Juan Carlos Erazo Álvarez

\section{Números antes-entre y después.}

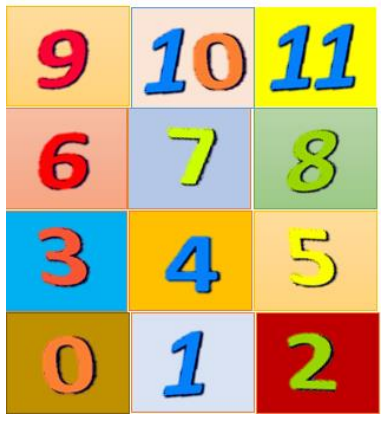

Figura 5. La rayuela y sus elementos.

Fuente. Elaboración propia

Para esta actividad tenemos que hacer que el niño se coloque en el número que está en la mitad de los tres casilleros y mencionar que a su derecha está el número después de y a la izquierda el número antes de. Al igual podemos enseñar el número entre, haciendo que coloque sus pies en el número de la izquierda y derecha.

\section{Canicas}

\section{Aprender las restas y contar elementos}

Hacer que el estudiante cuente las canicas, en este caso utilizaremos hasta el número 10, luego vamos a formar parejas y se lo podría realizar con el juego del barco de hunde, cuando ya estén formadas vamos a proceder a jugar lanzando las canicas dentro de un círculo previamente realizado ya sea con tiza o en la tierra directamente, luego a jugar, lanzando si golpea la canica del compañero se la ganan luego deben identificar cuantas canicas tuvieron al inicio y cuantas les quedo al final, así aplicamos la suma y resta. 
Universidad Nacional Experimental Francisco de Miranda (UNEFM). Santa Ana de Coro. Venezuela

Mayra Priscila Cáceres-Cabrera; Darwin Gabriel García-Herrera; Nancy Marcela Cárdenas-Cordero Juan Carlos Erazo Álvarez

\section{Noción dentro- fuera}

También se pude trabajar nociones dentro- fuera, realizando un agujero en la tierra y los niños deben formar una fila, a cierta distancia deben tratar de lanzar para que ingrese en el agujero. Hacemos que cuenten cuantas canicas ingresaron dentro del agujero y cuantas quedaron fuera.

Evaluar los resultados de aprendizaje obtenidos de los estudiantes. - Después de haber realizado cada uno de los pasos antes mencionado, el docente debe evaluar los resultados de los estudiantes obtenidos durante el juego, parte importante siendo fundamental para determinar si se logró los objetivos de los aprendizajes esperados. Evaluar los resultados ayudará a tomar una decisión adecuada para poder orientar a las necesidades de los estudiantes y si fue certero el haber aplicado ciertas estrategias lúdicas para enseñar las matemáticas.

\section{CONCLUSIONES}

Las actividades lúdicas son parte importante en la enseñanza durante la niñez puesto que son actividades que animan y atraen la atención de los niños y niñas, estas se debe aplicar en su desarrollo desde temprana edad hasta su adolecencia, ya que los juegos tradicionales son una estrategia que ayuda a desarrollar destrezas matemática de manera agradable y entretenida en donde los niños y niñas de inicial y preparatoria son los involucrados en el aprendizaje.

Las actividades lúdicas como juegos tradicionales son aplicables dentro del ámbito educativo es por eso que el docente debe ser muy precauteloso al emplearlos ya que al no ser aprovechados de la manera correcta, no tendrá ninguna validez para llegar a desarrollar las destrezas requeridas y sobre todo el estudiante perdería el interés por jugar o realizar estas actividades. 
Universidad Nacional Experimental Francisco de Miranda (UNEFM). Santa Ana de Coro. Venezuela

Mayra Priscila Cáceres-Cabrera; Darwin Gabriel García-Herrera; Nancy Marcela Cárdenas-Cordero Juan Carlos Erazo Álvarez

Existe aún deficiencia en el aprendizaje de los estudiantes debido a la falta de estrategias de enseñanza de los maestros no solo en el nivel inicial y preparatoria, es por eso importante que dentro de los juegos se implementen actividades que atraigan al estudiante y pueda fortalecer sus destrezas y habilidades mediante el juego ya que esto ayudará a su mejor desempeño escolar en los años posteriores de estudio.

\section{REFERENCIAS CONSULTADAS}

Awalludin , Y., Handoyo, E., \& Sri , S. (2018). Traditional Game on The Social Skill of Students in The Social Science Learning of Elementary School.[Juego tradicional sobre la habilidad social de los estudiantes en el aprendizaje de las ciencias sociales de la escuela primaria]. Journal of Primary Education, 7(2), 220-227. Recuperado el 30 de septiembre de 2020, de https://n9.cl/23gkm

Ferreira , L., \& Sousa, E. (2017). Juegos para enseñar matemáticas en la planificación para los profesores. [Games to teach math in planning for teachers early childhood education]. Asociación Colombiana de Matemática Educativa, 2(1), 1-11. Recuperado el 25 de septiembre de 2020, de https://n9.cl/k1zmz

González , A., Juan , M., \& Sánchez , M. (2014). La matemática nunca deja de ser un juego: investigaciones sobre los efectos del uso de juegos en la enseñanza de las matemáticas.[Mathematics never stops being a game: research on the effects of the use of games in the teaching of mathematics]. Educación Matemática, 26(3), 109-133. Recuperado el 4 de septiembre de 2020, de https://n9.cl/rr4kf

Morera , M. (2008). Generación tras generación, se recobran los juegos tradicionales.[Recovering traditional games generation after generation]. MHSalud, 5(1), 1-8. Obtenido de https://n9.cl/pz1oe

Perugachi , J. (2018). "Los juegos tradicionales acorde al origen de los estudiantes como estrategia para fomentar la interculturalidad, a traves de la asignatura de EEFF, en el tercer de E.B. paralelo "A" de la Escuela Municipal Cayambe, año lectivo 2017 - 2018".[Traditi]. Universidad Técnica del Norte. Obtenido de https://n9.cl/u4nk 
Universidad Nacional Experimental Francisco de Miranda (UNEFM). Santa Ana de Coro. Venezuela

Mayra Priscila Cáceres-Cabrera; Darwin Gabriel García-Herrera; Nancy Marcela Cárdenas-Cordero Juan Carlos Erazo Álvarez

Aristizábal , J., Colorado, H., \& Gutiérrez , H. (2016). El juego como una estrategia didáctica para desarrollar el pensamiento numérico en lascuatro operaciones básicas[Game as didactic strategy to develop numerical thought in the four basic operations]. Sophia, 117-125. Recuperado el 9 de Septiembre de 2020, de https://n9.cl/xvt2

Caballero , F., \& Espínola, J. (2016). El rechazo al aprendizaje de las matemáticas a causa de la violencia en el bachillerato tecnológico. [The rejection of learning mathematics due to violence in the technological high school]. Ra Ximhai, 12(3), 143-161. Obtenido de https://n9.cl/od5g

Carrillo, M., Padilla, J., Rosero, T., \& Villagómez, M. (2009). La motivación y el aprendizaje[Motivation and learning]. ALTERIDAD. Revista de Educación, 4(2), 20-32. Obtenido de https://n9.cl/6h3qw

Condor, T. (2019). Los juegos tradicionales como estrategia en el aprendizaje de la matemática en los estudiantes del segundo grado de primaria de la Institución Educativa $n^{\circ} 20326$ puquio cano-Hualmay, 2016[ Los juegos tradicionales como estrategia en el aprendizaje de la]. Universidad Nacional José Faustiño Sánchez Carrión.

El Universo. (26 de febrero de 2019). El Universo. Recuperado el 25 de octubre de 2020, de https://n9.cl/o1og

Fernández, A., Molina, V., \& Oliveras, M. (2016). Estudio de una propuesta lúdica para la educación científica y matemática globalizada en infantil. [Study of a playful proposal to globalized science and mathematics education in kindergarten]. Revista Eureka sobre Enseñanza y Divulgación de las Ciencias, 13(2), 1-12. Obtenido de https://n9.cl/n1rh

Garrote , D., Garrote, C., \& Jiménez , S. (2016). Factores Influyentes en Motivación y Estrategias de Aprendizaje en los Alumnos de Grado[Influential Factors in Motivation and Learning Strategies in Grade Students]. REICE, 14(2), 31-44. Obtenido de https://n9.cl/9xc3 
Universidad Nacional Experimental Francisco de Miranda (UNEFM). Santa Ana de Coro. Venezuela

Mayra Priscila Cáceres-Cabrera; Darwin Gabriel García-Herrera; Nancy Marcela Cárdenas-Cordero Juan Carlos Erazo Álvarez

Irvin , M. (2017). The Importance of Play in Early Childhood[La impotancia del juego en la primera infancia]. Northwestern College, lowa, 1-24. Obtenido de https://n9.cl/ayiu

Mendoza , M., Analuiza, E., \& Lara, L. (2017). Los juegos populares y su aporte didáctico en las clases de Educación Física [Popular games and their didactic contribution in Physical Education classes]. EmásF- Revista Digital de Educación Física, 8(44), 79-93. Obtenido de https://n9.cl/j9fv

Minerva, C. (2002). El juego: una estrategia importante.[Play is an important strategy]. Educere, 6(19), 289-296. Obtenido de https://n9.cl/8j1q

Moya , A. (2004). La matemática de los niños y niñas -Contribuyendo a la equidad[The girls and boys mathematics']. Sapiens. Revista Universitaria de Investigación, 5(2), 23-36. Obtenido de https://n9.cl/dg6i

Öfele, M. (2014). Juego, ternura y encuentro. Fundamentos en la primera infancia [Play, tenderness and encounter. Fundaments in early childhood]. Espacios en Blanco. Revista de Educación, 8(44), 79-93. Obtenido de https://n9.cl/aktae

Ospina , J. (2006). La motivación, motor del aprendizaje[Motivation, the Engine of Learning]. Revista Ciencias de la Salud, 4, 158-160. Obtenido de https://n9.cl/9r25

Putu , L., \& Prima, E. (2017). The Implementation of Traditional Games to Improve the Social Emotional Early Childhood [La implementación de juegos tradicionales para mejorar la primera infancia socioemocional]. Journal of Educational Science and Technology, 3(3), 178-184. Obtenido de https://n9.cl/th8ni

Quintanilla, N. (20016). Estrategias lúdicas dirigidas a la Enseñanza de la matemática a nivel de Educación Primaria.[Playful strategies aimed at the Teaching of mathematics at the Primary Education level]. Universida de Carabobo. Recuperado el 16 de septiembre de 2020, de https://n9.cl/gyrr

Restrepo, J., \& Tamayo , A. (2017). El juego como mediación pedagógica en la comunidad de una institución de protección, una experiencia llena de sentidos[Playing as a pedagogical mediation in the community of a protection institution, an experience full of meanings]. Revista Latinoamericana de Estudios Educativos, 13(1), 71-80. Obtenido de https://n9.cl/t104f 
Universidad Nacional Experimental Francisco de Miranda (UNEFM). Santa Ana de Coro. Venezuela

Mayra Priscila Cáceres-Cabrera; Darwin Gabriel García-Herrera; Nancy Marcela Cárdenas-Cordero Juan Carlos Erazo Álvarez

Sailema , Á. A., \& Sailema, M. (s.f.). Juegos tradicionales y populares del Ecuador. Publicaciones y Libros - Consejo Editorial UTA. Obtenido de https://n9.cl/r2hb

Sánchez, G. (2019). Estrategias Lúdicas en el aprendizaje de las matemáticas en los niños del primer año de EGB de la Unidad Educativa Darío Guevara.[Learning strategies in mathematics in children of the first year of EGB of the educational unit Dario Guevara.]. Universidad Tecnológica Indoamérica. Obtenido de https://n9.cl/tirn

Zafra, S., Vergel , M., \& Martínez, J. (2016). Ambiente de aprendizaje lúdico de las matemáticas para niños de la segunda infancia.[Environment of playfullness learning of mathematics for second childhood children]. Revista Logos, Ciencia \& Tecnología, 7(2), 14-22. Obtenido de https://n9.cl/niqg

(C2020 por los autores. Este artículo es de acceso abierto y distribuido según los términos y condiciones de la licencia Creative Commons Atribución-NoComercial-Compartirlgual 4.0 Internacional (CC BY-NC-SA 4.0) (https://creativecommons.org/licenses/by-nc-sa/4.0/) 